\title{
Evaluation and Comparison of Different Palatal Rugae Patterns between Dentulous and Edentulous Population of Mysuru City - A Preliminary Forensic Survey
}

\author{
Rabia Almasudi1 ${ }^{1}$ Ganesh Somashekarachar², Nagabhushana Doggalli3, \\ Meenakshi Srinivasa Iyer ${ }^{4}$, Sowmya Srinivas ${ }^{5}$, Anupama Aradhya ${ }^{6}$ \\ ${ }^{1}$ Department of Forensic Odontology, JSS Dental College and Hospital, JSSAHER, Mysore, \\ Karnataka, India, 2, 4, 5, 6 Department of Prosthodontics, Crown and Bridge, JSS Dental \\ College and Hospital, JSSAHER, Mysore, Karnataka, India, ${ }^{3}$ Department of Oral Medicine \\ and Radiology, JSS Dental College and Hospital, JSSAHER, Mysore, Karnataka, India.
}

\section{ABSTRACT}

\section{BACKGROUND}

Forensic odontology is a branch of dentistry which deals with appropriate handling and examination of dental evidences and with the proper evaluation and presentation of dental findings in the interest of justice. Identification of an individual is a prerequisite for certification of death and for personal, social and legal reasons. Palatal rugae are permanent and unique to each person, and can be used by clinicians and scientists to establish identity. It can be of special interest in edentulous cases and also in certain conditions, such as burned bodies or bodies that underwent severe decomposition. Thus the uniqueness, postmortem resistance, overall stability and additionally low utilization cost makes palatal rugae ideal forensic identification parameters. We wanted to compare the various palatal rugae patterns between dentate and edentulous subjects and also determine the comparison of the distribution of rugae number, length, shape, direction, unification between dentate and edentulous subjects.

\section{METHODS}

The study sample consisted of 60 subjects, 30 dentulous and 30 edentulous from JSS Dental College \& Hospital, Mysuru. The study group was equally divided between the sex, and was observed for number, length, shape, direction and unification of all palatal rugae.

\section{RESULTS}

The total number of rugae was higher in the dentulous group than in the edentulous group. In edentulous group, shorter lengths, lesser complexity rugae were seen compared to dentulous subjects. The total number of rugae on the right side in dentulous casts were $4.20 \pm 1.06$, while the total number on right side in Edentulous casts were $4.16 \pm 0.79$. On the left side mean total number of rugae in dentulous casts was $4.46 \pm 0.79$ whereas the mean total number of rugae in edentulous casts was 3.76 $\pm 0.77$

\section{CONCLUSIONS}

Palatal rugae pattern changed greatly after aging and loss of teeth regardless of time of edentulism and wearing denture.

\section{KEY WORDS}

Dentulous, Edentulous, Palatal Rugae, Forensic Identification
Corresponding Author:

Dr. Ganesh $S$,

Department of Prosthodontics,

Crown and Bridge, JSS Dental College and Hospital, JSSAHER, Mysore,

Karnataka, India.

E-mail: sganesh@jssuni.edu.in

DOI: $10.14260 / j e m d s / 2021 / 394$

How to Cite This Article:

Almasudi R, Somashekarachar G, Doggalli $N$, et al. Evaluation and comparison of different palatal rugae patterns between dentulous and edentulous population of Mysuru city - a preliminary forensic survey. J Evolution Med Dent Sci 2021;10(25): 1910-1916, DOI:

10.14260/jemds/2021/394

Submission 11-02-2021,

Peer Review 20-04-2021,

Acceptance 26-04-2021,

Published 21-06-2021.

Copyright (C) 2021 Rabia Almasudi et al. This is an open access article distributed under Creative Commons Attribution License [Attribution 4.0 International (CC $B Y$ 4.0)] 


\section{BACKGROUND}

Forensic odontology is a dental division that deals with the proper handling and analysis of dental evidences and the proper assessment and presentation of dental results in the interest of justice. ${ }^{1}$ Human identity, whether living or dead, is a cornerstone of humanity, and the identification of an unknown person has always been a matter of utmost importance to our culture. Human identification is based on scientific principles, mainly involving dental records, fingerprints and DNA comparisons. ${ }^{2}$ Palatal Rugoscopy i.e study of palatal rugae is the simple technique used by a forensic odontologist in human identification. ${ }^{3}$ Palatal rugae also called as rugae palatinae or plicae palatinae transverse refers to a series of transverse ridges on either side of the median palatal raphe and the incisive papillae, on the anterior portion of the palatal mucosa. ${ }^{4}$ Palatal Rugae are permanent and distinctive to each person and are stable, which can be used by clinicians and scientists to establish identity through discrimination. 5 Characteristics of palatal rugae such as uniqueness and stability make them convenient for forensic identification of victims. ${ }^{6}$

The special feature of palatal rugae being the soft tissue is their specific anatomic position within the oral cavity where they remain protected from high temperature or severe physical trauma. Therefore, known as the most protected morphologically individual soft tissue that remains preserved longer after death and is easily accessible over the entire lifetime. ${ }^{7}$ Palatal rugae have been equated with fingerprints and are unique to an individual. ${ }^{8}$ It can be of special interest in edentulous cases and also in certain conditions where there are no fingerprints to be studied, such as burned bodies or bodies that underwent severe decomposition. ${ }^{9}$ Rugae pattern may be specific to racial groups facilitating population identification. Thus the uniqueness, overall stability and additionally low utilization cost makes palatal rugae, ideal forensic identification parameters. ${ }^{10}$ In situations, where teeth is not present, for example complete edentulism, palatoscopy can be used. Palatal rugoscopy is relatively easy to obtain among the evidence taken from edentulous victims, which can be used for human identification. ${ }^{11}$ In this study we wanted to compare the various palatal rugae patterns between dentate and edentulous subjects and to evaluate and compare the distribution of rugae number, length, shape, direction, unification between dentate and edentulous subjects.

\section{METHODS}

A comparative, retrospective research study, consisting of 60 subjects in total which included patients visiting the Department of Prosthodontics, JSS Dental College and hospital, was conducted in January 2017 for a period of one month. The sample size was distributed equally among 30 dentates (15 males, 15 females) and 30 edentulous (15 males, 15 females) in the age group of $30-60$ years of population of Mysuru district. The sample size was selected to obtain statistically significant data. The criteria behind this sample selection was to compare variations among palatal rugae patterns between edentulous and dentulous subjects. The sample size was calculated using the formula.
$N=(Z \alpha)^{2} s^{2} / d^{2}$,

the $\mathrm{Z} \alpha$ values are 1.64 and 2.33 for $5 \%$ and $1 \%$ level of significance. The sample standard deviation was 5 and with a probability of $95 \%$, at an error rate of $5 \%$, the sample size estimated as $\mathrm{N}=(1.96)^{2}(5)^{2} / 2^{2}$ gives the sample of 24 subjects, if the allowance of $10 \%$ for attrition is assumed, then the corrected sample will be 27 subjects. Corrected sample size for $20 \%$ allowances is approximated to be 30 subjects in each group.

\section{Ethical Clearance}

Approval from the ethical committee of JSSAHER, Mysuru, was obtained to make the impression. The study was conducted in accordance with the ethical standards.

\section{Inclusion Criteria}

Healthy subjects, completely edentulous with atleast 1 year of edentulism and dentate subjects with complete permanent teeth.

\section{Exclusion Criteria}

- Subjects who underwent surgery

- Orthodontic treated subjects

- Subjects with traumatic injures of palate

- Subjects with bony or soft tissue protuberances.

\section{Impression}

After obtaining informed consent from the subjects diagnostic impressions of maxillary arch were made with irreversible hydrocolloid impression material (alginate) using sterilized perforated metal stock trays.

Impressions were poured with type 4 dental stone, vibrator was used to get void free cast and the base was prepared with Plaster of Paris according to manufacturer's instructions.

\section{Cast Analysis}

The casts were minutely observed with the help of a magnifying lens. A $0.5 \mathrm{~mm}$ black Graphite pencil marker was used to highlight the rugae patterns.

Thomas \& Kotze classification (Figs 1, 2 and 3) ${ }^{12}$ was followed for the interpretation of the rugae pattern in the cast. This classification includes number, length, shape, direction and unification of rugae. The entire rugae patterns were assessed and measured using caliper and scale

\section{Statistical Analysis}

The obtained data were entered into Microsoft excel sheet and results were obtained from recent version of the SPSS (Statistical package for social science) software. The descriptive statistic tests were performed to get frequencies, mean and standard deviation. For inferential statistics assuming the data follows the normal distribution. For comparing two groups, Independent sample $\mathrm{T}$ - test was used. For comparing frequencies between groups, chi-square test was used. 

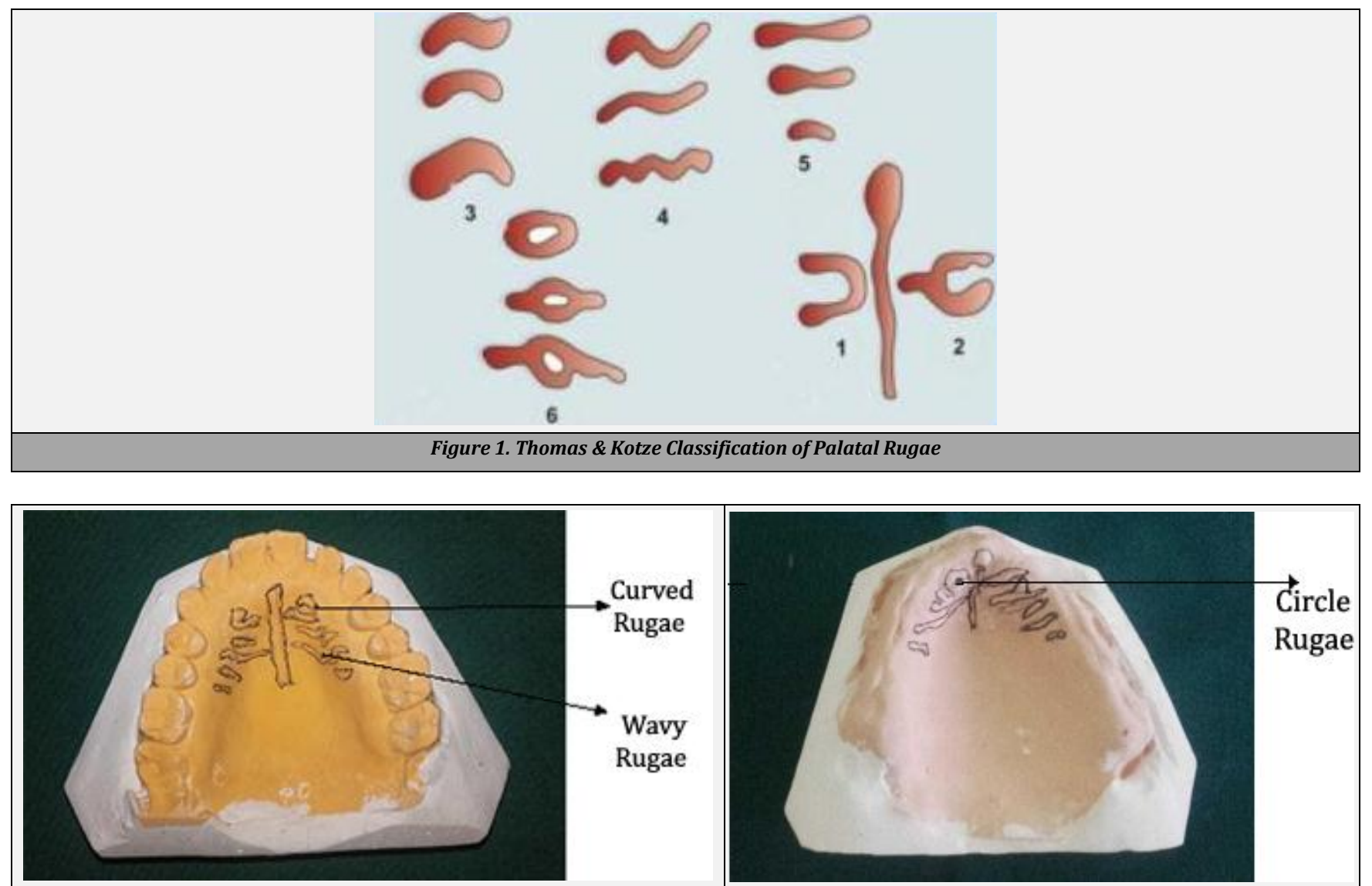

Circle Rugae
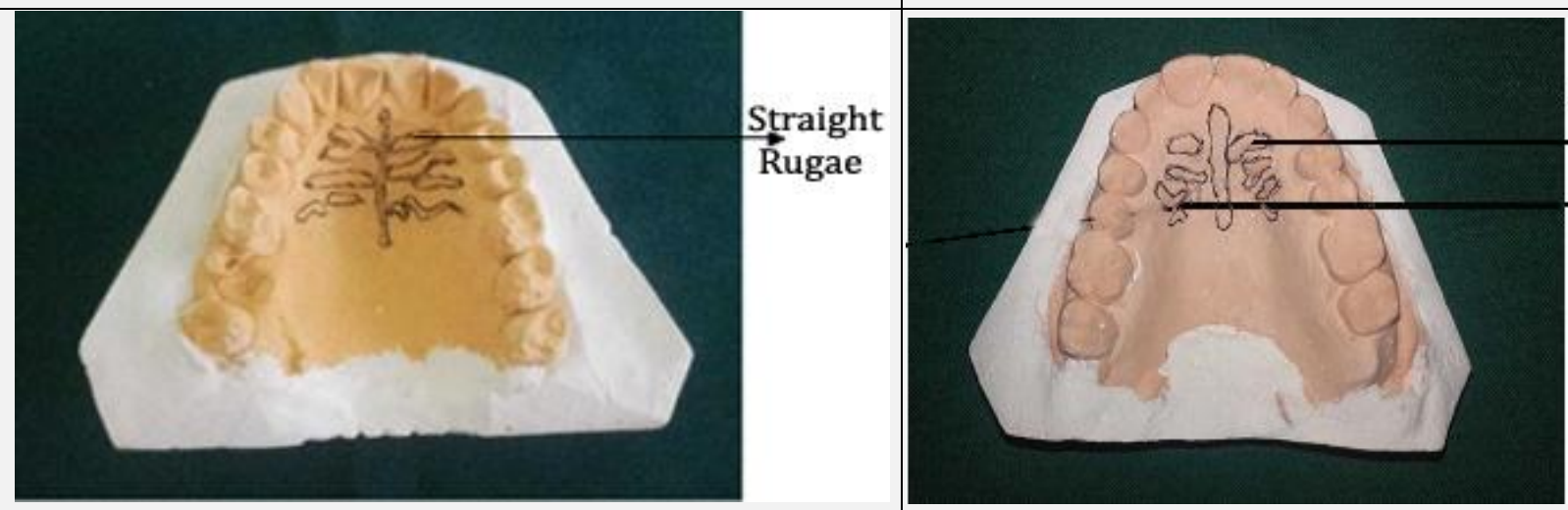

Backward

Rugae

Forward

Rugae

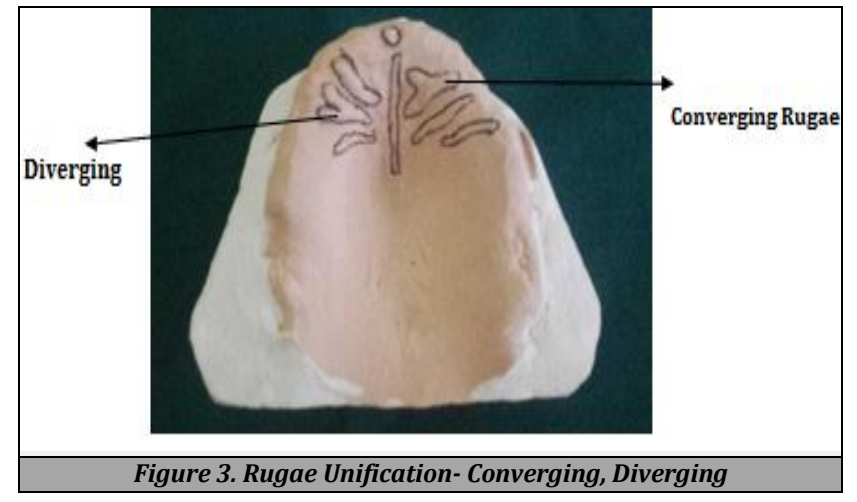

RESULTS

This study consisted of 60 subjects, 30 Dentulous and 30 edentulous subjects. Analysis of number, length, shape, direction, unification of rugae was done using Thomas \& Kotze classification. The mean age of the dentulous group was 37.30 +7.12 , whereas the mean age of the edentulous group was $52.53+6.69$.

Comparison of Number of Rugae between Dentulous and Edentulous Participants

In comparison with edentulous subjects, the total number of rugae was more in dentulous participants. The total number of rugae on the right side in dentulous casts were $4.20 \pm 1.06$, while the total number on right side in edentulous casts were $4.16 \pm 0.79$. On the left side mean total number of rugae in dentulous casts was $4.46 \pm 0.79$ whereas the mean total number of rugae in edentulous casts was $3.76 \pm 0.77$ Independent samples $t$ test revealed non significant difference in the total number of rugae on the right side $(\mathrm{t}=0.138, \mathrm{P}=$ 0.891). Significant difference in the total number of rugae on the left side $(t=3.60, P=0.01)$. 


\begin{tabular}{|lccccc|}
\hline \multirow{4}{*}{ Total_rugae_right } & Group & N & Mean & Std. Deviation & Std. Error Mean \\
\cline { 2 - 6 } Total_rugae_left & D & 30 & 4.2000 & 1.06350 & .19417 \\
& ED & 30 & 4.1667 & .79148 & .14450 \\
& ED & 30 & 4.4667 & .73030 & .13333 \\
\hline \multicolumn{5}{c}{ Table1. Comparison of Rugae between } \\
Dentulous and Edentulous Subjects \\
\hline
\end{tabular}

\section{Comparison of Rugae Pattern between Dentulous and Edentulous Casts Based on Length}

Primary rugae was the most predominant rugae followed by secondary and fragmentary rugae. The mean length of right primary rugae in dentulous cast was $3.26 \pm 0.63$ whereas in edentulous, it was $3.76 \pm 0.77$. In Independent samples $t$ test, length of primary rugae in dentulous and edentulous group revealed a significant difference $(t=2.728, P=0.008)$, The length of secondary rugae on right side of dentulous and edentulous casts revealed a significant difference $(\mathrm{t}=2.55, \mathrm{P}=$ $0.013)$. In-relation to the length of fragmentary rugae on right side in dentulous and edentulous groups, a non-significant difference was observed $(t=1.439, P=0.155)$. In Independent $t$-test, the length of primary rugae on left side in dentulous and edentulous groups revealed a non-significant difference $(\mathrm{t}=$ $0.394, P=0.695)$. Length of secondary rugae in dentulous and edentulous groups revealed a significant difference $(t=3.89, P$ $=0.001$ ). Length of left fragmentary rugae in dentulous and edentulous groups revealed significant difference $(t=2.80, P=$ 0.007).

\section{Comparison of Rugae Pattern between Dentulous and Edentulous Casts Based on Shape}

In total sample, wavy rugae pattern was the most predominant shape followed by curve, straight and circular. In dentulous and edentulous casts in relation to the shapes, on the right side - curve, wavy, straight and circular shapes showed nonsignificant difference whereas on the left side, a nonsignificant difference was observed in relation to curve and wavy shape, but straight shape had significant difference. $(\mathrm{t}=$ $2.99, \mathrm{P}=0.004$ )

\section{Comparison of Rugae Pattern between Dentulous and Edentulous Casts Based on Direction}

- On the right side in relation to direction of rugae forward, backward and perpendicular in dentulous and edentulous casts were non-significant.

- On left side forward and backward direction in dentulous and edentulous casts were non-significant but perpendicular direction revealed significant difference. $(\mathrm{t}$ $=3.261, \mathrm{P}=0.002$ )

\section{Comparison of Rugae Pattern between Dentulous and Edentulous Subjects Based on Unification}

- In both dentulous and edentulous casts on right side unification of rugae diverging and converging patterns were non-significant. On the left side, diverging pattern was noticed in both dentulous and edentulous casts which was significant with independent $\mathrm{t}$ test $\mathrm{t}=2.494, \mathrm{P}=$ 0.016 ).

- The total number of rugae in Dentulous subjects was more than in edentulous on both the sides. Whereas total number of rugae in dentulous subjects on left side was more than the right side. Total number of rugae in edentulous subjects on right side was more than the left side.

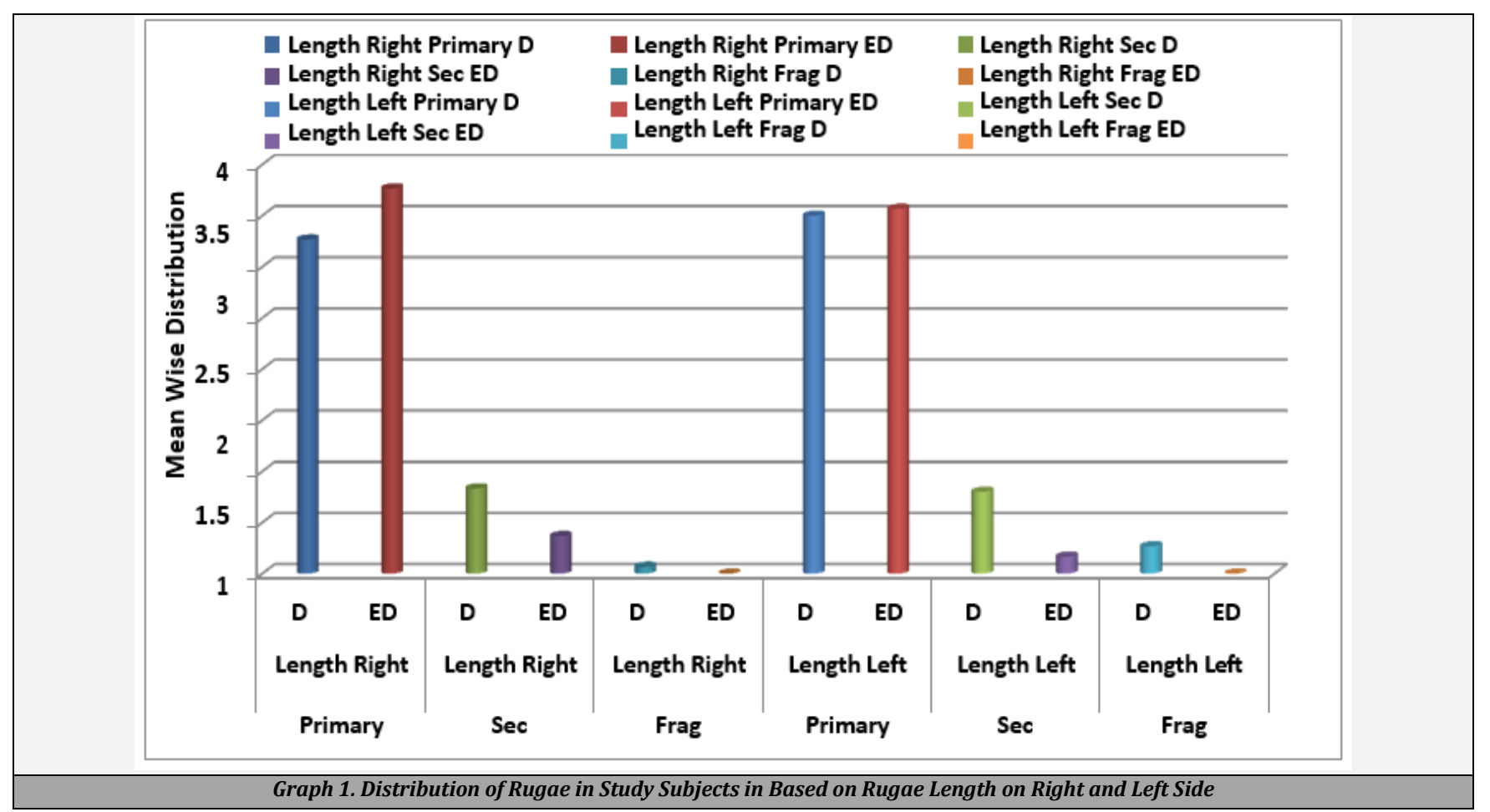




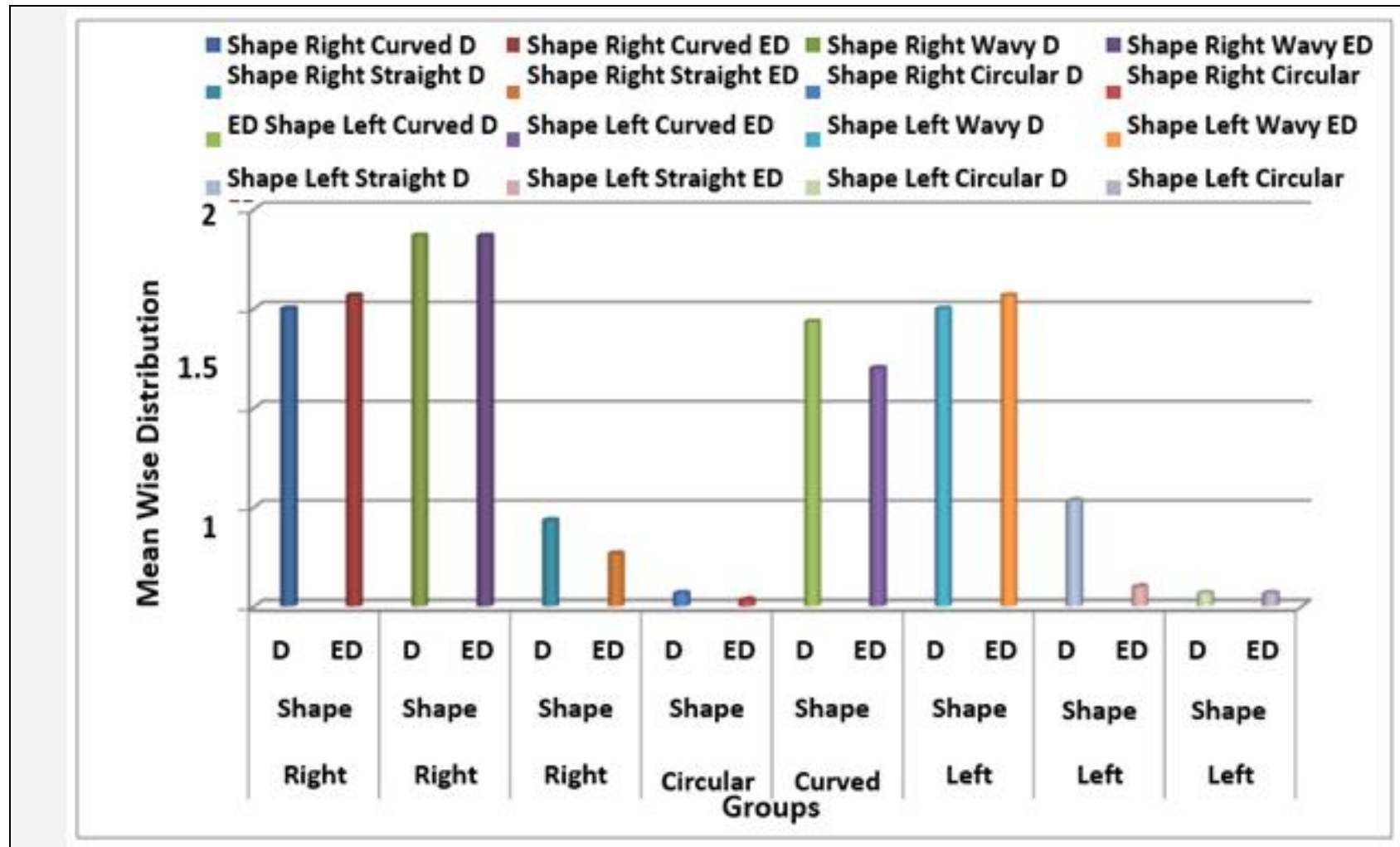

Graphs 2. Distribution of Rugae in Study Subjects Based on Shape in Right and Left Side

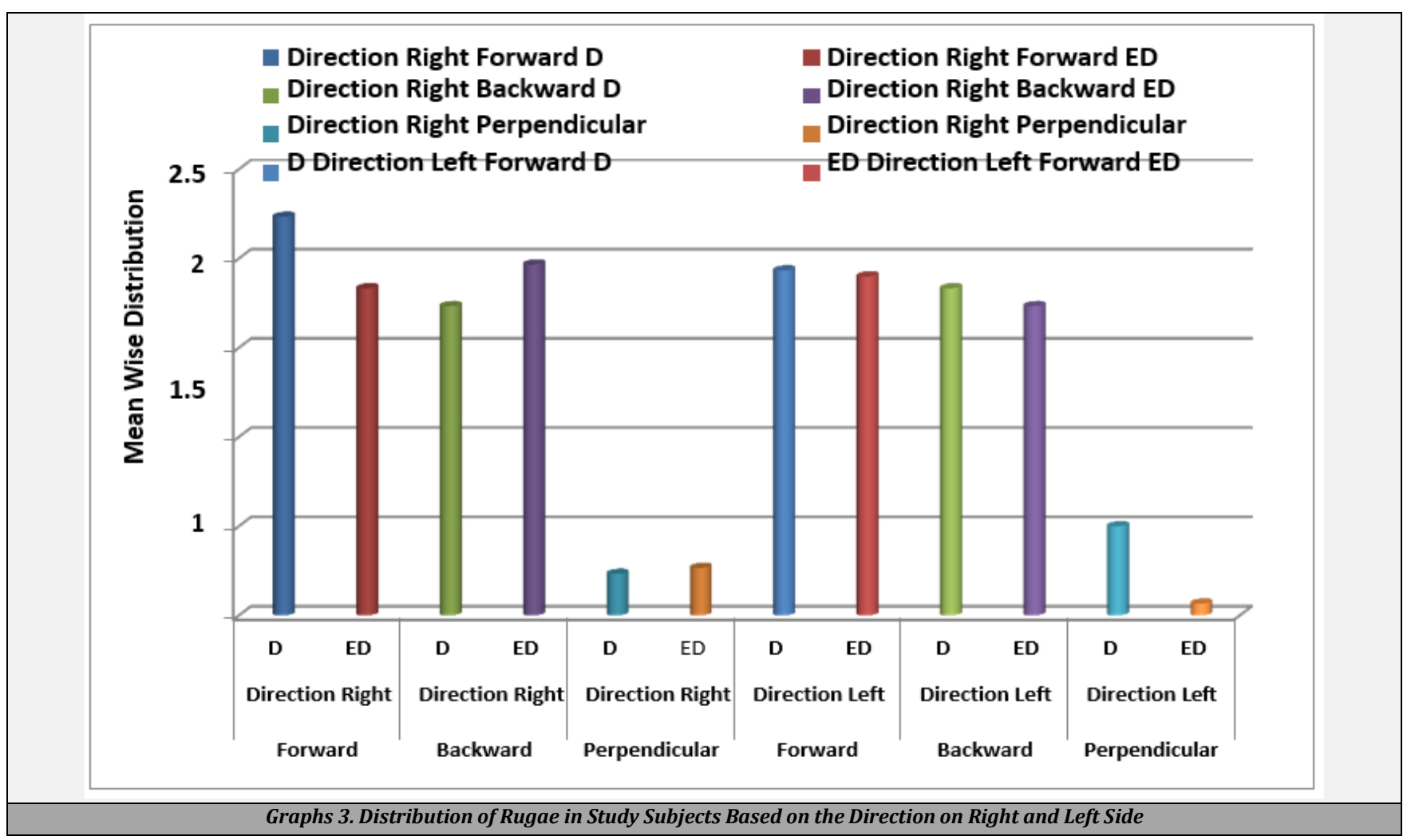

In the total sample, primary rugae was the most predominant rugae followed by secondary rugae and fragmentary rugae. Primary rugae was predominant on right side and left side in both dentulous and edentulous casts. Secondary rugae was predominant in dentulous. Secondary rugae was more on right side than left side. Rugae distribution was nearly equal on both the sides. Fragmentary rugae in dentulous was more than edentulous casts. Fragmentary rugae was predominant on left side in dentulous. In total sample, wavy rugae were more prominent in dentulous and edentulous casts followed by curved rugae, straight rugae and circular rugae. Wavy rugae were predominant on right side in dentulous and edentulous casts. Straight shape was predominant on left side in dentulous cast and on right side in edentulous cast. 


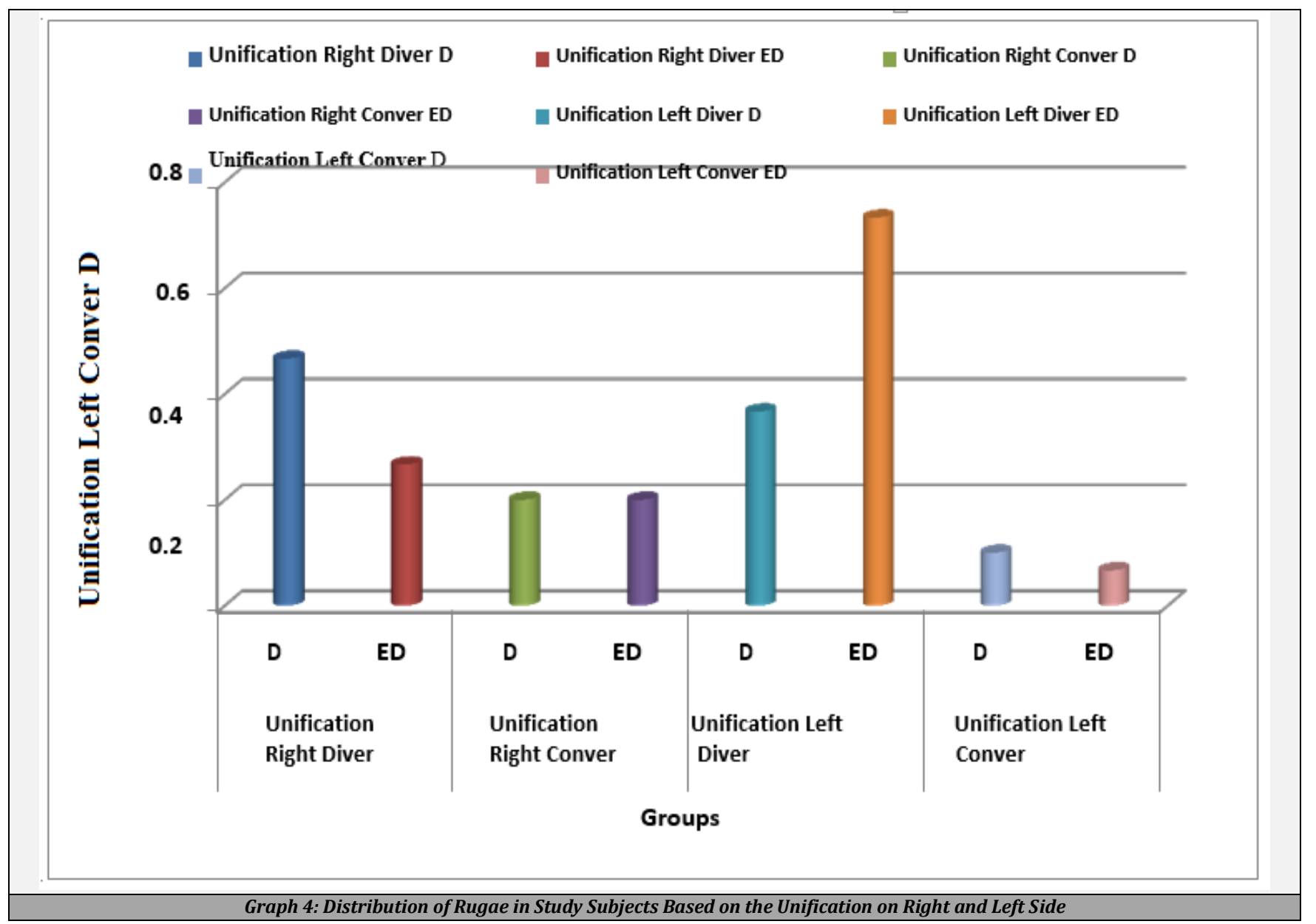

In the total sample, forward rugae were more prominent in both the dentulous and edentulous casts followed by backward rugae, and perpendicular rugae. Forward was predominant on right side in dentulous and left side in edentulous. Backward rugae and perpendicular rugae were predominant on left side in dentulous and on right side in edentulous. Of the total sample, diverging rugae were prominent in both the dentulous and edentulous casts followed by converging rugae. Diverging rugae were predominant on left side in edentulous and on right side in dentulous. Convergence was predominant on right side in both the dentulous and edentulous.

\section{DISCUSSION}

Rugae pattern have been studied for various purposes, including anthropology, genetics, forensic odontology, prosthodontics, and orthodontics. ${ }^{13}$ The uniqueness of rugae pattern in each individual and their unchanged nature during an individual's life makes them reliable parameters in forensic odontology. ${ }^{14}$

Despite the ongoing problem of quantitatively and qualitatively defining palatal scaffold patterns, they are unique to people and have a possible accurate identifying source. ${ }^{15}$

The current research showed that the total number of rugae and the number of main rugae in the edentulous group were substantially reduced and similar results were found by Lysell et al. ${ }^{16}$
In a study conducted in Iraq, 40 participants of whom 20 were dentate subjects and 20 were edentulous. Based on the effect of ageing on rugae, they observed significant reductions in the total number of rugae and primary rugae, while the number of secondary rugae remained approximately stable. The most common rugae shapes observed were wavy and curved forms as compared to straight and circular types. ${ }^{17}$ It is in agreement with our study. Curved, straight and circular were more frequent in dentate group, whereas wavy shapes were most prominent in edentulous group.

In a study done in Punjab, India, it was found that the most common shapes were wavy and curved, whereas straight and circular types were least common regardless of age. The edentulous group showed the highest means of curved pattern and total absence of circular pattern suggesting the presence of simpler form of rugae pattern. ${ }^{18}$ The results are in contrast with our study.

In the present study the total number of rugae in dentulous was more than in edentulous. Primary rugae were more in edentulous and secondary rugae in dentulous based on the length of the rugae. Our study is in agreement with Kapali et al, wherein they concluded that the length of rugae changes till 10 years of age thereafter remaining stable throughout life. ${ }^{18}$

On comparison of direction of rugae patterns, forward directed rugae were commonly observed both on the right and left side in dentulous groups, backward directed rugae pattern were more on the right side of edentulous and on left side of dentulous which was similar to a study done in Rajasthan state. ${ }^{19}$ Perpendicular rugae were more in dentulous in the present study in comparison with the study done by Bhatt $\mathrm{G}$ et 
al. ${ }^{19}$ where perpendicular rugae were more in edentulous group. ${ }^{17}$

Unification diverging rugae were more on the left side of edentulous group followed by right side of dentulous and converging rugae were more on right side of dentulous group. Ohtani et al. reported that features such as poorly demarcated rugae eminence and non-complex rugae patterns in edentulous patients were mainly due to the form of the edentulous palate itself and rarely due to dentures and could lead to difficulties in identifying specific points for personal identification. Continuous mechanical stimulation could lead to a great deal of morphological degeneration in the palatal mucosa involving rugae. ${ }^{20}$

\section{CONCLUSIONS}

Palatal rugae pattern changes were observed with aging regardless of time of edentulism and wearing of denture. The changes were reduced in number \& length. Less complex features were noted in edentulous subjects than young dentulous subjects.

Palatal rugae have been demonstrated during life to be strongly individualistic and consistent in form. It is well known that the palatal rugae pattern is specific to a human being, such as his fingerprint, so that palatal rugae could be used to identify an individual.

Data sharing statement provided by the authors is available with the full text of this article at jemds.com.

Financial or other competing interests: None.

Disclosure forms provided by the authors are available with the full text of this article at jemds.com.

\section{REFERENCES}

[1] Body identification guidelines. American Board of forensic odontology, Inc. J Am Den Assoc 1994;125(9):1244-50.

[2] Paliwal A, Wanjari S, Parwani R. Palatal rugoscopy establishing identity. J Forensic Dent Sci 2010;2(1):27-31.

[3] Bharath ST, Kumar GR, Dhanapal R, et al. Sex determination by discriminate function analysis of palatal rugae from a population of Coastal Andhra. J Forensic Dent Sci 2011;3(2):58-62.

[4] Amasaki H, Ogawa M, Nagasao J, et al. Distributional change of BrdU, PCNA, E2F1 and PAL31 molecules in developing murine palatal rugae. Ann Anat 2003;185(6):517-23.

[5] Patil MS, Patil SB, Acharya AB. Palatine rugae and their significance in clinical dentistry: a review of the literature. J Am Dent Assoc 2008;39(14):71-8.

[6] Shaughnessy PE. Introduction to forensic science. Dent Clin North Am 2001;45(2):217-27.

[7] Fahmi F, Al-Shamrani SM, Talic YF. Rugae pattern in a Saudi population sample of males and females. Saudi Dent J 2001:13(2):92-5.

[8] Almeida MA, Phillips C, Kula K, et al. Stability of palatal rugae as landmark for analysis of dental casts. Angle Orthod 1995;65(1):43-8.

[9] Caldas IM, Magalhaes T, Afonso A. Establishing identity using chieloscopy and palatoscopy. Forensic Sci Int 2007;165(1):1-9.

[10] Nayak P, Acharya AB, Padmini AT, et al. Differences in thepalatal rugae shape in two populations of India. Arch Oral Biol 2007;52(10):977-82.

[11] Filho IE, Sales-Peres SH, Sales-Peres A, et al. Palatal rugaepatterns as bioindicators for identification in forensic dentistry. RFO 2009;14(3):227-33.

[12] Thomas CJ, Kotze TJ. The palatal rugae pattern: a new classification. J Dent Assoc S Afr 1983;38(3):153-7.

[13] Jain S, Jain A. Rugoscopy as an adjuncent to sex differentiationin forensic odontology. Journal of Applied Dental and Medical Science 2015;1(1):1-6.

[14] Madhankumar S, Natarajan S, Maheswari U, et al. Palatal rugae for gender identification among selected population in Chennai India. Journal of Scientific Research and Reports 2013;2(2):491-6.

[15] Bhatt G. Comparison of rugae pattern between Dentulous and edentulous population of Rajasthan State. J Forensic Res 2015;6(1):1-3.

[16] Lysell L. Plicae palatinae transversae and papilla incisiva in man; a morphologic and genetic study. Acta Odontol Scand 1955;13(Suppl 18):5-137.

[17] Kapali S, Townsend G, Richards L, et al. Palatal rugae patterns in Australian aborigines and caucasians. Aust Dent J 1997;42(2):129-33.

[18] Caldas M, Magalhaes T, Afonso A. Establishing identity using cheiloscopy and palatoscopy. Forensic Sci Int 2007;165(1):1-9.

[19] Kaur P, Garg S, Sandhu S, et al. Comparison of palatal Rugae pattern between dentate and Edentate Punjabi population. International Journal in Physical and Applied Sciences 2015;2(2):31-40.

[20] Ohtani M, Nishida N, Chiba T, et al. Indication and limitation of using palatal rugae for personal identification in edentulous cases. Forensic Sci Int 2008;176(2-3):178-82. 